\title{
Hamiltonicity of Regular 2-Connected Graphs
}

H. J. Broersma

FACULTY OF APPLIED MATHEMATICS, UNIVERSITY OF TWENTE P.O. BOX 217, 7500 AE ENSCHEDE,

THE NETHERLANDS J. van den Heuvel*
DEPARTMENT OF MATHEMATICS AND STATISTICS,
SIMON FRASER UNIVERSITY
BURNABY, B.C., CANADA V5A 1S6

B. Jackson

DEPARTMENT OF MATHEMATICAL STUDIES, GOLDSMITHS' COLLEGE LONDON SE14 6NW, ENGLAND

\author{
H. J. Veldman \\ FACULTY OF APPLIED MATHEMATICS, \\ UNIVERSITY OF TWENTE \\ P.O. BOX 217, 7500 AE ENSCHEDE, \\ THE NETHERLANDS
}

\section{ABSTRACT}

Let $G$ be a $k$-regular 2-connected graph of order $n$. Jackson proved that $G$ is hamiltonian if $n \leq 3 k$. Zhu and Li showed that the upper bound $3 k$ on $n$ can be relaxed to $\frac{22}{7} k$ if $G$ is 3-connected and $k \geq 63$. We improve both results by showing that $G$ is hamiltonian if $n \leq \frac{7}{2} k-7$ and $G$ does not belong to a restricted class $\mathcal{F}$ of nonhamiltonian graphs of connectivity 2. To establish this result we obtain a variation of Woodall's Hopping Lemma and use it to prove that if $n \leq \frac{7}{2} k-7$ and $G$ has a dominating cycle (i.e., a cycle such that the vertices off the cycle constitute an independent set), then $G$ is hamiltonian. We also prove that if $n \leq 4 k-3$ and $G \notin \mathcal{F}$, then $G$ has a dominating cycle. For $k \geq 4$ it is conjectured that $G$ is hamiltonian if $n \leq 4 k$ and $G \notin \mathcal{F}$. (C) 1996 John Wiley \& Sons, Inc.

* Supported by a grant from the Natural Sciences and Engineering Research Council of Canada. Journal of Graph Theory Vol. 22, No. 2, 105-124 (1996)

(c) 1996 John Wiley \& Sons, Inc.

CCC 0364-9024/96/020105-20 


\section{INTRODUCTION AND MAIN RESULT}

We use [3] for terminology and notation not defined here and consider finite simple graphs only.

In [8], the following result was obtained.

Theorem 1 (Jackson [8]). Every 2-connected $k$-regular graph on at most $3 k$ vertices is hamiltonian.

As noted in [8], Theorem 1 is best possible for $k=3$ in view of the Petersen graph, and essentially best possible for $k \geq 4$. For future reference also, we define three classes $\mathcal{G}, \mathcal{H}$, and $\mathcal{J}$ of graphs illustrating the latter assertion. For a positive integer $p$, let $\mathcal{K}_{p}$ denote the set of all graphs consisting of three disjoint complete graphs, where each of the components has order at least $p$. Now $\mathcal{G}$ is the class of all spanning subgraphs of graphs that can be obtained as the join of $K_{2}$ and a graph in $\mathcal{K}_{1}$. The class $\mathcal{H}$ is the set of all spanning subgraphs of graphs that can be obtained from the join of $K_{1}$ and a graph $G$ in $\mathcal{K}_{2}$ by adding the edges of a triangle between three vertices from distinct components of $G$. The class $\mathcal{J}$ is the set of all spanning subgraphs of graphs that can be obtained from a graph $G$ in $\mathcal{K}_{3}$ by adding the edges of two triangles between two disjoint triples of vertices, each containing one vertex of each component of $G$. It is easy to check that all graphs in $\mathcal{G} \cup \mathcal{H} \cup \mathcal{J}$ are nonhamiltonian. (Indeed, $\mathcal{G}, \mathcal{H}$, and $\mathcal{J}$ were first obtained by Watkins and Mesner [12] in a characterization of the 2-connected graphs which have three vertices which are not contained in a common cycle.) Furthermore, each of the classes $\mathcal{G}$, $\mathcal{H}$, and $\mathcal{J}$ contains 2 -connected $k$-regular graphs on $3 k+4$ vertices for even $k \geq 4$, and $3 k+5$ vertices for all $k \geq 3$. (Note that $\mathcal{G}, \mathcal{H}$, and $\mathcal{J}$ are not pairwise disjoint.) We set $\mathcal{F}=\mathcal{G} \cup \mathcal{H} \cup \mathcal{J}$.

Theorem 1 has been extended in several papers, e.g., in [2], [7], and [15]. The strongest among these extensions is due to Hilbig [7]. Let $\Pi$ denote the Petersen graph and $\Pi^{\Delta}$ the 3-regular graph obtained from $\Pi$ by replacing one vertex by a triangle.

Theorem 2 (Hilbig [7]). Let $G$ be a 2-connected $k$-regular graph on at most $3 k+3$ vertices. Then $G$ is hamiltonian if and only if $G \notin\left\{\Pi, \Pi^{\Delta}\right\}$.

In [9], the following improvement of Theorem 1 for 3-connected graphs is conjectured. (Note that no graph in $\mathcal{F}$ is 3 -connected.)

Conjecture 1. (JACKSON, LI, and ZHU [9]). For $k \geq 4$, every 3-connected $k$-regular graph on at most $4 k$ vertices is hamiltonian.

Conjecture 1 is a special case of Häggkvist's conjecture, appearing in [8], that every $m$ connected $k$-regular graph $(k \geq 4)$ on at most $(m+1) k$ vertices is hamiltonian. However, for $k \equiv 0(\bmod 4)$, the graph $\overline{K_{k}} \vee\left(\overline{K_{k-1}}+2 K_{k+1}\right)$ contains a nonhamiltonian $\frac{1}{2} k$-connected $k$-regular spanning subgraph $G_{k}$, showing that Häggkvist's conjecture is not true in general. The graphs $G_{k}$ were independently found by $H$. A. Jung and the third author. For a more detailed description we refer to [9] or [10]. The graphs $G_{k}$ also show that Conjecture 1 would be best possible.

A first step toward proving Conjecture 1 was made in [9]. A cycle $C$ of a graph $G$ is called a dominating cycle if $V(G) \backslash V(C)$ is an independent set of $G$.

Theorem 3 (Jackson, Li, and Zhu [9]). Let $G$ be a 3-connected $k$-regular graph on at most $4 k$ vertices. Then for $k \geq 63$, every longest cycle of $G$ is a dominating cycle. 
In the graph $G_{k}$, every longest cycle is dominating. Still Theorem 4 is essentially best possible: for even $k \geq 8$, the graph $K_{3} \vee\left(2 K_{k}+2 K_{k+1}\right)$ of order $4 k+5$ has a 3-connected $k$-regular spanning subgraph containing no dominating cycle.

In [14], Theorem 3 was used to obtain another result in the direction of Conjecture 1.

Theorem 4 (Zhu and Li [14]). For $k \geq 63$, every 3-connected $k$-regular graph on at most $\frac{22}{7} k$ vertices is hamiltonian.

Our main result is the following.

Theorem 5. Let $G$ be a 2-connected $k$-regular graph on at most $\frac{7}{2} k-7$ vertices. Then $G$ is hamiltonian if and only if $G \notin \mathcal{F}$.

Since no graph in $\mathcal{F}$ is 3-connected, the following improvement of Theorem 4 is an immediate consequence of Theorem 5 .

Corollary. Every 3-connected $k$-regular graph on at most $\frac{7}{2} k-7$ vertices is hamiltonian.

The necessity of the condition for hamiltonicity in Theorem 5 is obvious. The sufficiency is an immediate consequence of the following two results.

Theorem 6. Let $G$ be a $k$-regular graph on at most $\frac{7}{2} k-7$ vertices. If $G$ contains a dominating cycle, then $G$ is hamiltonian.

Theorem 7. Let $G$ be a 2 -connected $k$-regular graph on at most $4 k-3$ vertices. Then $G$ contains a dominating cycle or $G \in \mathcal{F}$.

Theorem 6 is proved in Section 3. The proof uses a variation of Woodall's Hopping Lemma [13], which is the subject of Section 2. The proof of Theorem 7, which is based on ideas from [5] and [11], is given in Section 4.

Our proof of Theorem 5 (via Theorems 6 and 7) uses several ideas from [4], where a relatively short proof of (an extension of) Theorem 1 occurs. In particular the idea of breaking the proof into two parts in the way reflected by Theorems 6 and 7, stems from [4].

In view of the above results we propose a strengthening of Conjecture 1 .

Conjecture 2. Let $G$ be a 2 -connected $k$-regular graph on at most $4 k$ vertices. Then for $k \geq 4, G$ is hamiltonian if and only if $G \notin \mathcal{F}$.

\section{A HOPPING LEMMA}

We first develop some additional terminology and notation.

Let $C$ be a cycle of a graph $G$. We call $C$ extendable if there exists an extension of $C$, i.e., a cycle $C^{\prime}$ with $V(C) \subseteq V\left(C^{\prime}\right)$ and $V(C) \neq V\left(C^{\prime}\right)$. For $v \in V(G) \backslash V(C)$, the cycle $C$ is $v$-extendable if there exists a $v$-extension of $C$, i.e., an extension with vertex set $V(C) \cup\{v\}$.

We denote by $\vec{C}$ the cycle $C$ with a given orientation, and by $\bar{C}$ the cycle $C$ with the reverse orientation. If $u, v \in V(C)$, then $u \vec{C} v$ denotes the consecutive vertices of $C$ from $u$ to $v$ in the direction specified by $\vec{C}$. The same vertices, in reverse order, are given by 
$v \overleftarrow{C} u$. We will consider $u \vec{C} v$ and $v \overleftarrow{C} u$ both as paths and as vertex sets. We use $u^{+}$to denote the successor of $u$ on $\vec{C}$ and $u^{-}$to denote its predecessor. If $Z \subseteq V(C)$, then $Z^{+}=\left\{z^{+} \mid z \in Z\right\}$ and $Z^{-}=\left\{z^{-} \mid z \in Z\right\}$. Similar notation is used for paths. When more than one cycle or path is under consideration, we write $u^{+C}, u^{-C}$ instead of just $u^{+}, u^{-}$ in order to avoid ambiguity.

If $A$ and $B$ are subsets of $V(G)$, then $\varepsilon(A, B)$ denotes the number of edges with one end in $A$ and the other in $B$; the edges with both ends in $A \cap B$ are counted twice. For $a \in V(G)$, we write $\varepsilon(a, B)$ instead of $\varepsilon(\{a\}, B)$.

In our variation of Woodall's Hopping Lemma [13], Lemma 8 below, we use the following hypotheses and definitions.

Let $G$ be a graph, $\vec{C}$ a cycle of $G$ with $V(C) \neq V(G)$, and $a$ a vertex in $V(G) \backslash V(C)$. Assume $C$ is not $a$-extendable. Set

$$
X_{1}=N(a) \cap V(C)
$$

and for $i \geq 1$,

$$
\begin{gathered}
Y_{i}=X_{i}^{+} \cap X_{i}^{-}, \\
U_{i}=X_{i}^{+} \backslash Y_{i}, \\
W_{i}=X_{i}^{-} \backslash Y_{i} \\
X_{i+1}^{\prime}=\left\{v \in V(C) \mid \text { there exist six neighbors } w_{1}, u_{1}, w_{2}, u_{2}, w_{3}, u_{3} \text { of } v\right. \\
\text { such that } \left.w_{j} \in W_{i}, u_{j} \in U_{i} \text { and } w_{j}^{+} \vec{C} u_{j}^{-} \subseteq X_{i} \cup Y_{i}(j=1,2,3)\right\}, \\
X_{i+1}^{\prime \prime}=N\left(Y_{i}\right) \cap V(C), \\
X_{i+1}=X_{i} \cup X_{i+1}^{\prime} \cup X_{i+1}^{\prime \prime} .
\end{gathered}
$$

Then $X_{1} \subseteq X_{2} \subseteq \cdots$ and $Y_{1} \subseteq Y_{2} \subseteq \cdots$. Set

$$
\begin{aligned}
& X=\bigcup_{i=1}^{\infty} X_{i}, \\
& Y=\bigcup_{i=1}^{\infty} Y_{i} .
\end{aligned}
$$

Then

$$
N(Y) \cap V(C) \subseteq X
$$

The height $h(x)$ of $x \in X$ is defined by

$$
h(x)=\min \left\{i \mid x \in X_{i}\right\} .
$$

A path $P=x_{1} \vec{P} x_{2}$ is called a hopping path if each of the following conditions is satisfied:

$$
x_{1}, x_{2} \in X
$$




$$
V(P)=V(C)
$$

if $1 \leq i<\max \left\{h\left(x_{1}\right), h\left(x_{2}\right)\right\}$ and $y \in Y_{i} \backslash\left\{x_{1}, x_{2}\right\}$,

$$
\text { then }\left\{y^{-P}, y^{+P}\right\}=\left\{y^{-C}, y^{+C}\right\}
$$

if $1 \leq i<\max \left\{h\left(x_{1}\right), h\left(x_{2}\right)\right\}$, then $X_{i} \backslash\left\{x_{1}, x_{2}\right\}$

$$
\text { contains at most one vertex } x \text { for which }\left\{x^{-P}, x^{+P}\right\} \neq\left\{x^{-C}, x^{+C}\right\} \text {. }
$$

The height $h(P)$ of a hopping path $P=x_{1} \vec{P} x_{2}$ is defined by

$$
h(P)=\max \left\{h\left(x_{1}\right), h\left(x_{2}\right)\right\} .
$$

Note that our definitions differ from those given in [13] in that:

-we do not require $N(a) \subseteq V(C)$;

—we add the sets $X_{i}^{\prime}$ to $X$;

-our conditions (4) and (5) for a hopping path are more restrictive.

Lemma 1. There exists no hopping path.

Proof. Assuming there exists a hopping path, let $P=x_{1} \vec{P} x_{2}$ be one such that

$$
h(P) \text { is minimum, and }
$$

$$
\text { subject to }(6), h\left(x_{1}\right)+h\left(x_{2}\right) \text { is minimum. }
$$

Assume without loss of generality that $h\left(x_{1}\right) \geq h\left(x_{2}\right)$. We have $h(P) \neq 1$, otherwise $x_{1}, x_{2} \in X_{1}=N(a) \cap V(C)$ and $x_{1} \vec{P} x_{2} a x_{1}$ is an $a$-extension of $C$, contradicting our assumptions. Hence $h\left(x_{1}\right)=h(P) \geq 2$. Set $i=h\left(x_{1}\right)-1$, so that $x_{1} \in X_{i+1} \backslash X_{i}$. We derive contradictions in two cases.

Case 1. $h\left(x_{2}\right)<h\left(x_{1}\right)$.

We distinguish two subcases (not necessarily mutually exclusive).

Case 1.1. $x_{1} \in X_{i+1}^{\prime} \backslash X_{i}$.

By (5) and the definition of $X_{i+1}^{\prime}$ there exists at least one pair $w, u$ with $w \in W_{i}, u \in$ $U_{i}, w^{+} \vec{C} u^{-} \subseteq X_{i} \cup Y_{i}$ and $w x_{1}, u x_{1} \in E(G)$ such that $w \vec{C} u$ contains neither $x_{2}$ nor a vertex $x \in X_{i} \backslash\left\{x_{2}\right\}$ with $\left\{x^{-P}, x^{+P}\right\} \neq\left\{x^{-C}, x^{+C}\right\}$. It follows that $w \vec{C} u$ is a subpath of $P$. We have

$$
u, w \notin X_{i}
$$

otherwise $u \vec{C} u^{-C}$ or $w^{+C} \vec{C} w$ is a hopping path of height at most $i$, contradicting (6). In particular, $u, w \neq x_{2}$. Suppose $u \in x_{1} \vec{P} w$. (The case $w \in x_{1} \vec{P} u$ is completely analo-

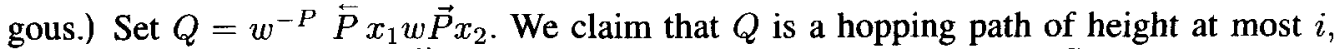
contradicting (6). Since $w \vec{C} u$ is a subpath of $P$, we have $w^{-P}=w^{+C} \in X_{i}$, and so $Q$ satisfies (2). Since $P$ satisfies (3) and $V(Q)=V(P), Q$ satisfies (3). We now establish (4) and (5) for $Q$. Since (4) and (5) hold for $P$ and $\left\{v^{-Q}, v^{+Q}\right\}=\left\{v^{-P}, v^{+P}\right\}$ for each internal vertex $v$ of $Q$ with $v \neq x_{1}, w$, it suffices to show that $x_{1}, w \notin X_{i-1} \cup Y_{i-1}$ if $i \geq 2$. Since $x_{1} \in X_{i+1} \backslash X_{i}, x_{1} \notin X_{i-1}$. Also, $x_{1} \notin Y_{i-1}$, otherwise $w \in X_{i}$, contradicting (8). 
Since $w \notin X_{i}$, certainly $w \notin X_{i-1}$. Finally, $w \notin Y_{i-1}$, otherwise $x_{1} \in X_{i}$. Furthermore, $h(Q)=\max \left\{h\left(w^{-P}\right), h\left(x_{2}\right)\right\} \leq i$.

Case 1.2. $x_{1} \in X_{i+1}^{\prime \prime} \backslash X_{i}$.

Then there exists a vertex $y \in Y_{i} \backslash Y_{i-1}$ with $x_{1} y \in E(G)$. We have

$$
y \notin X_{i}
$$

otherwise $y^{+C} \vec{C} y$ would be a hopping path of height at most $i$. In particular, $y \neq x_{2}$. By (4), $y^{-P} \in X_{i}$. Set $Q=y^{-P} \stackrel{\leftarrow}{P} x_{1} y \vec{P}_{x_{2}}$. We obtain a contradiction to (6) by showing that $Q$ is a hopping path of height at most $i$. It suffices to show that $x_{1}, y \notin X_{i-1} \cup Y_{i-1}$ if $i \geq 2$ (as in Case 1.1). Clearly, $x_{1} \notin X_{i-1}$. Also, $x_{1} \notin Y_{i-1}$, otherwise $y \in X_{i}$, contradicting (9). Since $y \notin X_{i}$, certainly $y \notin X_{i-1}$. Finally, $y \in Y_{i} \backslash Y_{i-1}$, so $y \notin Y_{i-1}$.

Case 2. $h\left(x_{2}\right)=h\left(x_{1}\right)(=i+1)$.

Again we distinguish two subcases.

Case 2.1. $x_{1} \in X_{i+1}^{\prime} \backslash X_{i}$ (or $x_{2} \in X_{i+1}^{\prime} \backslash X_{i}$ ).

Define $w$ and $u$ as in Case 1.1. We have

$$
u, w \notin X_{i+1},
$$

otherwise $u \vec{C} u^{-C}$ or $w^{+C} \vec{C} w$ is a hopping path that contradicts (6) or (7). In particular, $u, w \neq x_{2}$. Assume for example that $w \in x_{1} \vec{P} u$ and set $Q=u^{-P} \overleftarrow{P} x_{1} u \vec{P} x_{2}$. Now $Q$ is a hopping path of height $i+1$ that contradicts (7). To see this, it suffices to show that $x_{1}, u \notin X_{i} \cup Y_{i}$. Clearly, $x_{1} \notin X_{i}$. Also, $x_{1} \notin Y_{i}$, otherwise the hopping path $x_{1}^{+C} \vec{C} x_{1}$ contradicts (7). By (10), $u \notin X_{i}$. Finally, $u \notin Y_{i}$ by definition of $U_{i}$.

Case 2.2. $x_{1}, x_{2} \in X_{i+1}^{\prime \prime} \backslash X_{i}$.

Then there exist vertices $y_{1}, y_{2} \in Y_{i} \backslash Y_{i-1}$ with $x_{1} y_{1}, x_{2} y_{2} \in E(G)$. We have

$$
y_{j} \notin X_{i+1} \quad(j=1,2),
$$

otherwise $y_{j}^{+C} \vec{C} y_{j}$ is a hopping path that contradicts (6) or (7). Now $y_{1}^{-P} \stackrel{\leftarrow}{P} x_{1} y_{1} \vec{P} y_{2} x_{2}$ $\bar{P} y_{2}^{+P}$ (if $y_{1} \in x_{1} \vec{P} y_{2}$ ) or $y_{2}^{-P} \bar{P} x_{1} y_{1} \bar{P} y_{2} x_{2} \bar{P} y_{1}^{+P}$ (if $y_{2} \in x_{1} \vec{P} y_{1}$ ) is a hopping path of height at most $i$, contradicting (6). To see this, it suffices to show that $x_{1}, x_{2}, y_{1}, y_{2} \notin$ $X_{i-1} \cup Y_{i-1}$ if $i \geq 2$. Clearly, $x_{1}, x_{2} \notin X_{i-1}$ and $y_{1}, y_{2} \notin Y_{i-1}$. Also, $x_{1}, x_{2} \notin Y_{i-1}$, otherwise $y_{1} \in X_{i}$ or $y_{2} \in X_{i}$, contradicting (11). Finally, by (11), $y_{1}, y_{2} \notin X_{i-1}$.

We shall now briefly indicate how Lemma 1 will be used to prove Theorem 6 . In the remainder of this section we first apply Lemma 1 to obtain several other lemmas concerning the existence of edges with one end in $X^{+} \cup X^{-}$.

In Section 3 we suppose that $G$ is a nonhamiltonian graph satisfying the hypotheses of Theorem 6. We apply the lemmas of this section to a nonextendable dominating cycle in $G$, in order to restrict the number of edges between $X^{+} \cup X^{-}$and $V(G) \backslash X$. The regularity condition then implies that there must be "many" edges between $X^{+} \cup X^{-}$and $X$. We then obtain a contradiction to $k$-regularity by showing that this number of edges is greater than $k|X|$.

Lemma 2. $X \cap X^{+}=\emptyset$. 
Proof. Suppose $X \cap X^{+}$contains a vertex $x$. Then $x \vec{C} x^{-}$is a hopping path, contradicting Lemma 1.

Set

$$
U=X^{+} \backslash Y, \quad W=X^{-} \backslash Y
$$

\section{Lemma 3.}

(a) $U$ and $W$ are independent sets.

(b) If $z_{1}, z_{2} \in U$ or $z_{1}, z_{2} \in W$, then there exists no pair of vertices $v, v^{+} \in z_{1} \vec{C} z_{2}$ such that $z_{1} v^{+}, z_{2} v \in E(G)$.

(c) If $u \in U$ and $w \in W$, then there exists no pair of vertices $v, v^{+} \in w^{+} \vec{C} u^{-}$such that $u v, w v^{+} \in E(G)$ or $u v^{+}, w v \in E(G)$.

(d) If $x \in X, v \in(U \cup W) \backslash\left\{x^{+}, x^{-}\right\}$and $x v \in E(G)$, then $N\left(x^{+}\right) \cap v^{+} \vec{C} x^{-} \cap(U \cup W)=$ $N\left(x^{-}\right) \cap x^{+} \vec{C}^{-} \cap(U \cup W)=\emptyset$.

Proof. (a) Suppose $u_{1}, u_{2} \in U$ and $u_{1} u_{2} \in E(G)$. By definition of $U$ and by Lemma 2, $u_{1}, u_{2} \notin X \cup Y$. But then $u_{1}^{-} \overleftarrow{C} u_{2} u_{1} \vec{C} u_{2}^{-}$is a hopping path, contradicting Lemma 1 . Hence $U$, and similarly $W$, is an independent set.

(b) Suppose $z_{1}, z_{2} \in U, v, v^{+} \in z_{1} \vec{C} z_{2}$ and $z_{1} v^{+}, z_{2} v \in E(G)$. By (a), $v \neq z_{1}$ and $v^{+} \neq z_{2}$. As in the proof of (a), $z_{1}, z_{2} \notin X \cup Y$. Hence by (1), $v, v^{+} \notin Y$. By Lemma 2, at most one of the vertices $v$ and $v^{+}$is in $X$. We conclude that $z_{1}^{-} \bar{C} z_{2} v \bar{C} z_{1} v^{+} \vec{C} z_{2}^{-}$is a hopping path. This contradiction proves one half of (b). The other half is proved similarly.

(c) Suppose $u \in U, w \in W, v, v^{+} \in w^{+} \vec{C} u^{-}$and either $u v, w v^{+} \in E(G)$ or $u v^{+}, w v \in$ $E(G)$. Arguing as in the proof of (b) we have $u, w \notin X \cup Y, v, v^{+} \notin Y$ and $\left|\left\{v, v^{+}\right\} \cap X\right| \leq 1$. It follows that $u^{-} \bar{C} v^{+} w \overleftarrow{C} u v \bar{C} w^{+}$(if $u v, w v^{+} \in E(G)$ ) or $u^{-} \bar{C} v^{+} u \vec{C} w v \bar{C} w^{+}$(if $\left.u v^{+}, w v \in E(G)\right)$ is a hopping path, a contradiction.

(d) This is an immediate consequence of (a), (b), and (c).

Set

$$
t=|U|(=|W|)
$$

and

$$
S=V(C) \backslash(X \cup Y)
$$

For $Z \subseteq V(G)$ define

$$
e(Z)=\varepsilon(U \cup W, Z)
$$

For $z, z^{\prime} \in V(G)$ we write $e(z)$ instead of $e(\{z\})$ and $e\left(z, z^{\prime}\right)$ instead of $e\left(\left\{z, z^{\prime}\right\}\right)$.

\section{Lemma 4.}

(a) If $x \in X$ and $x^{+} \in U$, then $e\left(x^{+}\right) \leq t-\frac{1}{2} e(x)+1$. If $x \in X$ and $x^{-} \in W$, then $e\left(x^{-}\right) \leq t-\frac{1}{2} e(x)+1$.

(b) If $x \in X, x^{+} \in U, x^{-} \in W$ and $N(x) \cap\left((U \cup W) \backslash\left\{x^{+}, x^{-}\right\}\right) \neq \emptyset$, then $e\left(x^{+}, x^{-}\right) \leq$ $t-\frac{1}{2} e(x)+2$.

(c) If $v, v^{+} \in S$, then $e\left(v, v^{+}\right) \leq t+3$. 
Proof. (a) It suffices to prove the first half, the second is symmetric. Assume $x \in X$ and $x^{+} \in U$. If $N(x) \cap(U \cup W) \subseteq\left\{x^{+}, x^{-}\right\}$, then we are done, since $e\left(x^{+}\right) \leq t$ by Lemma 6(a). In the remaining case, let $p$ be the first vertex of $N(x) \cap(U \cup W)$ on $x^{++} \vec{C} x^{--}$and set

$$
\begin{array}{ll}
a_{1}=\left|U \cap x^{+} \vec{C} p\right|, & a_{2}=\left|U \cap p \vec{C} x^{-}\right|, \\
b_{1}=\left|W \cap x^{+} \vec{C} p\right|, & b_{2}=\left|W \cap p \vec{C} x^{-}\right| .
\end{array}
$$

Then $b_{1}+b_{2}=t+|W \cap\{p\}|, e(x) \leq 1+a_{2}+b_{2}$ and by Lemma 3(a) and (d), $e\left(x^{+}\right) \leq b_{1}$. Hence

$$
e\left(x^{+}\right)+\frac{1}{2} e(x) \leq b_{1}+\frac{1}{2}\left(1+a_{2}+b_{2}\right)
$$

We complete the proof of (a) by showing that the right-hand side of (12) is at most $t+1$. If $p \in U$, then $a_{2}=b_{2}$ and hence

$$
b_{1}+\frac{1}{2}\left(1+a_{2}+b_{2}\right)=b_{1}+b_{2}+\frac{1}{2}=t+\frac{1}{2} .
$$

If $p \in W$, then $a_{2}=b_{2}-1$ and hence

$$
b_{1}+\frac{1}{2}\left(1+a_{2}+b_{2}\right)=b_{1}+b_{2}=t+1 \text {. }
$$

(b) Let $x$ satisfy the stated conditions. First assume $e(x)=3$. Let $p$ be the unique vertex in $N(x) \cap\left((U \cup W) \backslash\left\{x^{+}, x^{-}\right\}\right)$and assume without loss of generality that $p \in U$. Using Lemma 3(a) and (d) we obtain

$$
\begin{aligned}
e\left(x^{+}, x^{-}\right)+\frac{1}{2} e(x) & \leq\left|W \cap x^{+} \vec{C} p^{-}\right|+\left|U \cap p \vec{C} x^{-}\right|+\frac{3}{2} \\
& =\left|U \cap x^{+} \vec{C} p^{-}\right|+\left|U \cap p \vec{C} x^{-}\right|+\frac{3}{2}=t+\frac{3}{2}<t+2 .
\end{aligned}
$$

Now assuming $e(x) \geq 4$, let $p$ be the first and $q$ the last vertex of $N(x) \cap(U \cup W)$ on $x^{++} \vec{C} x^{--}$. Set

$$
\begin{array}{ll}
a_{1}=\left|U \cap x^{+} \vec{C} p\right|, & a_{2}=|U \cap p \vec{C} q|, \quad a_{3}=\left|U \cap q \vec{C} x^{-}\right| \\
b_{1}=\left|W \cap x^{+} \vec{C} p\right|, \quad b_{2}=|W \cap p \vec{C} q|, \quad b_{3}=\left|W \cap q \vec{C} x^{-}\right| .
\end{array}
$$

Then $b_{1}+b_{2}+b_{3}=t+|W \cap\{p, q\}|, e(x) \leq 2+a_{2}+b_{2}$ and by Lemma 3(a) and (d), $e\left(x^{+}, x^{-}\right) \leq b_{1}+a_{3}$. Hence

$$
e\left(x^{+}, x^{-}\right)+\frac{1}{2} e(x) \leq b_{1}+a_{3}+1+\frac{1}{2}\left(a_{2}+b_{2}\right)
$$

We complete the proof of (b) by showing that the right-hand side of (13) is at most $t+2$. If $p, q \in U$, then $a_{2}=b_{2}+1, a_{3}=b_{3}$ and hence

$$
b_{1}+a_{3}+1+\frac{1}{2}\left(a_{2}+b_{2}\right)=b_{1}+b_{2}+b_{3}+\frac{3}{2}=t+\frac{3}{2} .
$$

The case $p, q \in W$ is symmetric. If $p \in U, q \in W$, then $a_{2}=b_{2}, a_{3}=b_{3}-1$ and hence

$$
b_{1}+a_{3}+1+\frac{1}{2}\left(a_{2}+b_{2}\right)=b_{1}+b_{2}+b_{3}=t+1 \text {. }
$$


Finally, if $p \in W, q \in U$, then $a_{2}=b_{2}, a_{3}=b_{3}$ and hence

$$
b_{1}+a_{3}+1+\frac{1}{2}\left(a_{2}+b_{2}\right)=b_{1}+b_{2}+b_{3}+1=t+2 .
$$

(c) Assume $v, v^{+} \in S$. We distinguish two cases.

Case 1. $N(v) \cap W=\emptyset$ or $N\left(v^{+}\right) \cap U=\emptyset$.

Assume without loss of generality that $N(v) \cap W=\emptyset$. If $N(v) \cap U=\emptyset$, then $e\left(v, v^{+}\right)=$ $e\left(v^{+}\right) \leq t+2$, otherwise $v^{+}$would belong to $X_{i}^{\prime}$ for some $i \geq 2$. Thus we may assume $N(v) \cap U \neq \emptyset$. Let $p$ be the first vertex of $N(v) \cap U$ on $v^{+} \vec{C} v$. Since $N(v) \cap W=\emptyset$, we have $N(v) \cap v^{+} \vec{C} p^{-} \cap(U \cup W)=\emptyset$. By Lemma 3(b), (c) we have $N\left(v^{+}\right) \cap p^{+} \vec{C} v \cap(U \cup W)=\emptyset$. Also, $\left|N\left(v^{+}\right) \cap(U \cup W) \cap v^{+} \vec{C} p\right| \leq \frac{1}{2}\left|(U \cup W) \cap v^{+} \vec{C} p\right|+2$, otherwise $v^{+}$would belong to $X$. We conclude that

$$
\begin{aligned}
e\left(v, v^{+}\right) & \leq|U \cap p \vec{C} v|+\frac{1}{2}\left|(U \cup W) \cap v^{+} \vec{C} p\right|+2 \\
& =|U \cap p \vec{C} v|+\left|U \cap v^{+} \vec{C} p\right|+2=t+3
\end{aligned}
$$

Case 2. $N(v) \cap W \neq \emptyset$ and $N\left(v^{+}\right) \cap U \neq \emptyset$.

Let $p$ be the last vertex of $N(v) \cap W$ on $v^{+} \vec{C} v$ and $q$ the first vertex of $N\left(v^{+}\right) \cap U$ on $v^{+} \vec{C} v$. (Possibly, $p=v^{+}$and/or $q=v$.) Using Lemma 3(b), (c) we obtain

$$
\begin{gathered}
q \in p \vec{C} v \\
\left(N(v) \cup N\left(v^{+}\right)\right) \cap U \subseteq q \vec{C} v, \\
\left(N(v) \cup N\left(v^{+}\right)\right) \cap W \subseteq v^{+} \vec{C} p .
\end{gathered}
$$

Furthermore, by Lemma 3(b),

$$
\begin{gathered}
\varepsilon\left(\left\{v, v^{+}\right\}, U \cap q \vec{C} v\right) \leq|U \cap q \vec{C} v|+1 \\
\varepsilon\left(\left\{v, v^{+}\right\}, W \cap v^{+} \vec{C} p\right) \leq\left|W \cap v^{+} \vec{C} p\right|+1
\end{gathered}
$$

By (14), addition of the inequalities in (15) yields

$$
\begin{aligned}
e\left(v, v^{+}\right) & \leq|U \cap q \vec{C} v|+\left|W \cap v^{+} \vec{C} p\right|+2 \\
& =|W \cap q \vec{C} v|+\left|W \cap v^{+} \vec{C} p\right|+3 \leq|W|+3=t+3
\end{aligned}
$$

Let $Z_{U}$ be the set of all vertices $v \in S$ such that $v^{+} \in S \cap N\left(u_{1}\right)$ and $v^{-} \in S \cap N\left(u_{2}\right)$ for some $u_{1}, u_{2} \in U$ with $v \in u_{1} \vec{C} u_{2}$. Define $Z_{W}$ analogously.

\section{Lemma 5.}

(a) If $u_{1}, u_{2} \in U$, then $\varepsilon\left(\left\{u_{1}, u_{2}\right\}, S\right) \leq|S|$. If $w_{1}, w_{2} \in W$, then $\varepsilon\left(\left\{w_{1}, w_{2}\right\}, S\right) \leq|S|$.

(b) If $u_{1}, u_{2}, u_{3} \in U$, then $\varepsilon\left(\left\{u_{1}, u_{2}, u_{3}\right\}, S\right) \leq|S|+t+\left|Z_{U}\right|$. If $w_{1}, w_{2}, w_{3} \in W$, then $\varepsilon\left(\left\{w_{1}, w_{2}, w_{3}\right\}, S\right) \leq|S|+t+\left|Z_{W}\right|$.

(c) If $z \in Z_{U}$ or $z \in Z_{W}$, then $\varepsilon(z, S) \leq \frac{1}{2}|S|$. 
Proof. We only prove the assertions concerning $U$; those concerning $W$ follow similarly.

(a) Let $u_{1}, u_{2} \in U$ and let $S^{\prime}$ be the vertex set of one of the components of $C-(X \cup Y)$. We can assume, without loss of generality, that $S^{\prime} \subseteq u_{2} \vec{C} u_{1}$. Let $u$ be the unique vertex in $U \cap S^{\prime}$ and $w$ the unique vertex in $W \cap S^{\prime}$. Now define

$$
\begin{aligned}
& S_{1}=\left\{v \in S^{\prime} \mid u_{1} v \in E(G)\right\}, \\
& S_{2}=\left\{v \in S \backslash\{w\} \mid u_{2} v^{+} \in E(G)\right\} .
\end{aligned}
$$

By Lemma 3(b) we have $S_{1} \cap S_{2}=\emptyset$. Using Lemma 3(a) this gives

$$
\varepsilon\left(\left\{u_{1}, u_{2}\right\}, S^{\prime}\right)=\left|S_{1}\right|+\left|S_{2}\right| \leq\left|S^{\prime}\right| .
$$

Summing over all components of $C-(X \cup Y)$ we obtain $\varepsilon\left(\left\{u_{1}, u_{2}\right\}, S\right) \leq|S|$.

(b) Let $u_{1}, u_{2}, u_{3} \in U$ and let $S^{\prime}$ be the vertex set of one of the components of $C$ $(X \cup Y)$. We can assume, without loss of generality, that $u_{2} \in u_{1} \vec{C} u_{3}$ and $S^{\prime} \subseteq u_{3} \vec{C} u_{1}$. Let $u$ be the unique vertex in $U \cap S^{\prime}$ and $w$ the unique vertex in $W \cap S^{\prime}$. Now define

$$
\begin{aligned}
& S_{1}=\left\{v \in\left(S^{\prime} \cup\left\{w^{+}\right\}\right) \backslash\{u\} \mid u_{1} v^{-} \in E(G)\right\}, \\
& S_{2}=\left\{v \in S^{\prime} \mid u_{2} v \in E(G)\right\} \\
& S_{3}=\left\{v \in S^{\prime} \backslash\{w\} \mid u_{3} v^{+} \in E(G)\right\} .
\end{aligned}
$$

By Lemma 3(b) we have $S_{1} \cap S_{2}=S_{2} \cap S_{3}=\emptyset$. Also, by definition, $S_{1} \cap S_{3} \subseteq Z_{U}$. Using Lemma 3(a) this gives

$$
\varepsilon\left(\left\{u_{1}, u_{2}, u_{3}\right\}, S^{\prime}\right)=\left|S_{1}\right|+\left|S_{2}\right|+\left|S_{3}\right| \leq\left|S^{\prime}\right|+1+\left|Z_{U} \cap S^{\prime}\right| .
$$

Summing over all components of $C-(X \cup Y)$ we obtain $\varepsilon\left(\left\{u_{1}, u_{2}, u_{3}\right\}, S\right) \leq|S|+t+\left|Z_{U}\right|$.

(c) Let $z \in Z_{U}$ and suppose $u_{1}, u_{2} \in U$ such that $z \in u_{1} \vec{C} u_{2}, z^{+} \in S \cap N\left(u_{1}\right)$ and $z^{-} \in S \cap N\left(u_{2}\right)$. By Lemma 3(b), $\varepsilon(z, U)=0$. Furthermore, there exists no pair of vertices $v, v^{+} \in S$ such that $z v, z v^{+} \in E(G)$, otherwise there would exist a hopping path. For example, if $v, v^{+} \in S \cap u_{2} \vec{C} u_{1}$ and $z v, z v^{+} \in E(G)$, then $u_{1}^{-} \bar{C} v^{+} z v \bar{C} u_{2} z^{-} \bar{C} u_{1} z^{+} \vec{C} u_{2}^{-}$ is a hopping path. We conclude that $\varepsilon(z, S) \leq \frac{1}{2}|S|$.

Recall that so far $C$ was only assumed not to be $a$-extendable. In our next lemma we make a stronger assumption about $C$. Set

$$
R=V(G) \backslash(V(C) \cup\{a\})
$$

Lemma 6. Assume $C$ is not extendable. If $z_{1}, z_{2} \in X^{+}$or $z_{1}, z_{2} \in X^{-}$, then there exists no $\left(z_{1}, z_{2}\right)$-path with all internal vertices in $R$.

Proof. Assume $C$ is not extendable and, without loss of generality, $z_{1}, z_{2} \in X^{+}$. Suppose $z_{1}$ and $z_{2}$ are joined by a path with all internal vertices in $R$. Set $G^{\prime}=G+z_{1} z_{2}$. Since $C$ is not extendable in $G, C$ is not $a$-extendable in $G^{\prime}$. Define $X^{\prime}, Y^{\prime}$, and $U^{\prime}$ for $G^{\prime}$ in the same way as $X, Y$, and $U$ were defined for $G$. Clearly, $X \subseteq X^{\prime}$. Hence by Lemma 2 (applied to $\left.G^{\prime}\right), z_{1}, z_{2} \notin X^{\prime}$. Since $z_{1} z_{2} \in E\left(G^{\prime}\right)$ and $N_{G^{\prime}}\left(Y^{\prime}\right) \cap V(C) \subseteq X^{\prime}$, it also follows from Lemma 2 that $z_{1}, z_{2} \notin Y^{\prime}$. But then $z_{1}, z_{2} \in U^{\prime}$, contradicting Lemma 3(a). 
We finally note that, mutatis mutandis, the above results obviously remain valid if, instead of just a vertex $a$ outside $C$, a subset $A$ of $V(G)$ is considered such that $A \cap V(C)=\emptyset$ and $A$ induces a connected subgraph of $G$. The condition that $C$ is not a-extendable is then replaced by the requirement that there exists no extension of $C$ whose vertex set is contained in $V(C) \cup A$, while $X_{1}$ is defined as $N(A) \cap V(C)$ and $R$ as $V(G) \backslash(V(C) \cup A)$.

\section{PROOF OF THEOREM 6}

Using the results of Section 2 we now prove Theorem 6.

Proof of Theorem 6. Let $G$ be a $k$-regular graph of order $n$ such that $G$ contains a dominating cycle and

$$
n \leq \frac{7}{2} k-7 \text {. }
$$

By Dirac's Theorem [6], $G$ is hamiltonian if $n \leq 2 k$. Thus we may assume

$$
k \geq 6
$$

Assuming $G$ is nonhamiltonian, let $\vec{C}$ be a nonextendable dominating cycle of $G$ and $a$ be a vertex in $V(G) \backslash V(C)$. Define $X, Y, U, W, t, S, Z_{U}, Z_{W}, R$ and the function $e: 2^{V(G)} \rightarrow \mathbb{N}$ as in Section 2. Additionally define

$$
\begin{aligned}
X_{Y Y} & =\left\{v \in X \mid v^{-}, v^{+} \in Y\right\}, \\
X_{W Y} & =\left\{v \in X \mid v^{-} \in W, v^{+} \in Y\right\}, \\
X_{Y U} & =\left\{v \in X \mid v^{-} \in Y, v^{+} \in U\right\}, \\
X_{W U} & =\left\{v \in X \mid v^{-} \in W, v^{+} \in U\right\}, \\
X_{0} & =\left\{v \in X_{W U} \mid e(v)=2\right\} .
\end{aligned}
$$

Set $x=|X|, y=|Y|, s=|S|, r=|R|, x_{Y Y}=\left|X_{Y Y}\right|, x_{W Y}=\left|X_{W Y}\right|, x_{Y U}=\left|X_{Y U}\right|$, $x_{W U}=\left|X_{W U}\right|$ and $x_{0}=\left|X_{0}\right|$.

We make a few observations that will be used repeatedly. Clearly,

$$
\begin{gathered}
x \geq|N(a) \cap V(C)|=|N(a)|=k, \\
t=x-y
\end{gathered}
$$

and by (16),

$$
s=n-r-x-y-1 \leq \frac{7}{2} k-r-x-y-8 .
$$

By Lemma 6 and the fact that $C$ is a dominating cycle,

$$
\varepsilon(Y \cup\{a\}, R) \leq r .
$$

Hence by (1) and the fact that $G$ is $k$-regular,

$$
\varepsilon(Y \cup\{a\}, X) \geq k(y+1)-r .
$$


Let $X^{\prime}$ be a subset of $X$, of cardinality $x^{\prime}$. Clearly,

$$
\varepsilon\left(Y \cup\{a\}, X \backslash X^{\prime}\right) \leq\left(x-x^{\prime}\right)(y+1) .
$$

Hence by (21),

$$
\varepsilon\left(Y \cup\{a\}, X^{\prime}\right) \geq\left(k-x+x^{\prime}\right)(y+1)-r,
$$

implying that

$$
e\left(X^{\prime}\right) \leq k x^{\prime}-\left(k-x+x^{\prime}\right)(y+1)+r .
$$

Since $x_{Y U}=x_{W Y}=y-x_{Y Y}$, we have

$$
x_{W U}=x-x_{Y Y}-x_{Y U}-x_{W Y}=x-2 y+x_{Y Y},
$$

implying that

$$
x_{Y Y}=2 y-x+x_{W U} \geq 2 y-x .
$$

We now distinguish several cases and subcases and derive contradictions in each of them.

Case 1. $t \geq 5$.

Using Lemma 4(a), (b), (c) and observing that $x_{W Y}=x_{Y U}=t-x_{W U}$ we obtain

$$
\begin{aligned}
2 e(S)= & 2 \sum_{v \in S} e(v)=\sum_{v \in X_{W U} \backslash X_{0}} e\left(v^{-}, v^{+}\right)+\sum_{v \in X_{0}} e\left(v^{-}\right)+\sum_{v \in X_{0}} e\left(v^{+}\right) \\
& +\sum_{v \in X_{W Y}} e\left(v^{-}\right)+\sum_{v \in X_{Y}} e\left(v^{+}\right)+\sum_{v, v^{+} \in S} e\left(v, v^{+}\right) \\
\leq & \sum_{v \in X_{W U \backslash X_{0}}}\left(t-\frac{1}{2} e(v)+2\right)+2 x_{0} t+\sum_{v \in X_{W Y}}\left(t-\frac{1}{2} e(v)+1\right) \\
& +\sum_{v \in X_{Y U}}\left(t-\frac{1}{2} e(v)+1\right)+(s-t)(t+3)=\left(x_{W U}-x_{0}\right)(t+2) \\
& +2 x_{0} t+2\left(t-x_{W U}\right)(t+1)+(s-t)(t+3)-\frac{1}{2} e\left(X \backslash\left(X_{0} \cup X_{Y Y}\right)\right) \\
= & t\left(t-x_{W U}+x_{0}+s-1\right)-2 x_{0}+3 s-\frac{1}{2} e\left(X \backslash\left(X_{0} \cup X_{Y Y}\right)\right) .
\end{aligned}
$$

By (1), $e(Y)=0$ and by Lemma $6, e(R) \leq 2 r$. Hence by (25),

$$
\begin{aligned}
e(X)= & 2 t k-e(S)-e(R) \geq \frac{1}{2} t\left(4 k-t+x_{W U}-x_{0}-s+1\right) \\
& +x_{0}-\frac{3}{2} s-2 r+\frac{1}{4} e\left(X \backslash\left(X_{0} \cup X_{Y Y}\right)\right) .
\end{aligned}
$$

Since

$$
\begin{aligned}
e(X) & =e\left(X_{0}\right)+e\left(X_{Y Y}\right)+e\left(X \backslash\left(X_{0} \cup X_{Y Y}\right)\right) \\
& =2 x_{0}+e\left(X_{Y Y}\right)+e\left(X \backslash\left(X_{0} \cup X_{Y Y}\right)\right),
\end{aligned}
$$

(26) implies

$$
e\left(X_{Y Y}\right)+\frac{3}{4} e\left(X \backslash\left(X_{0} \cup X_{Y Y}\right)\right) \geq \frac{1}{2} t\left(4 k-t+x_{W U}-x_{0}-s+1\right)-x_{0}-\frac{3}{2} s-2 r
$$


On the other hand, by (22),

$$
\begin{aligned}
& e\left(X_{Y Y}\right)+\frac{3}{4} e\left(X \backslash\left(X_{0} \cup X_{Y Y}\right)\right) \\
& =\frac{1}{4} e\left(X_{Y Y}\right)+\frac{3}{4} e\left(X \backslash X_{0}\right) \leq \frac{1}{4}\left(k x_{Y Y}-\left(k-x+x_{Y Y}\right)(y+1)+r\right) \\
& \quad+\frac{3}{4}\left(k\left(x-x_{0}\right)-\left(k-x_{0}\right)(y+1)+r\right)=\frac{1}{4}\left(k\left(x_{Y Y}+3 x-3 x_{0}\right)\right. \\
& \left.\quad-\left(4 k-x+x_{Y Y}-3 x_{0}\right)(y+1)+4 r\right) .
\end{aligned}
$$

Combining (27) and (28) we obtain

$$
\begin{aligned}
& 2 t\left(4 k-t+x_{W U}-x_{0}-s+1\right)-4 x_{0}-6 s-8 r \\
& \quad \leq k\left(x_{Y Y}+3 x-3 x_{0}\right)-\left(4 k-x+x_{Y Y}-3 x_{0}\right)(y+1)+4 r .
\end{aligned}
$$

By (20), the inequality (29) remains valid if we replace $s$ by $\frac{7}{2} k-r-x-y-8$. Doing so and eliminating $t$ and $x_{W U}$ via (19) and (23), we obtain

$$
\begin{aligned}
& x_{0}(2 x+y-3 k+7)-x_{Y Y}(2 x-y-k+1)-2 x^{2}+3 x y \\
& \quad+2 k x-3 k y-2 r x+2 r y-23 x+12 y+17 k+6 r-48 \geq 0 .
\end{aligned}
$$

Since $t \geq 5$ by hypothesis, we have

$$
-2 r x+2 r y+6 r=-2 r t+6 r \leq-4 r \leq 0 .
$$

Hence by (30),

$$
\begin{aligned}
& x_{0}(2 x+y-3 k+7)-x_{Y Y}(2 x-y-k+1)-2 x^{2}+3 x y+2 k x \\
& \quad-3 k y-23 x+12 y+17 k-48 \geq 0 .
\end{aligned}
$$

Case 1.1. $2 x+y-3 k+7>0$.

Clearly, $x_{0} \leq x_{W U}$. Hence by (23), in (31) we may replace $x_{0}$ by $x-2 y+x_{Y Y}$ to obtain

$$
-2 x_{Y Y}(k-y-3)-2 y^{2}-k x+3 k y-16 x-2 y+17 k-48 \geq 0 .
$$

Case 1.1.1. $y \leq \frac{1}{2} k$.

Then by (17), $k-y-3 \geq 0$. Hence by (32),

$$
-2 y^{2}-k x+3 k y-16 x-2 y+17 k-48 \geq 0 .
$$

Since $y \leq \frac{1}{2} k$, the left-hand side of (33) is an increasing function of $y$. Hence in (33) we may replace $y$ by $\frac{1}{2} k$ to obtain

$$
-k(x-k)-16(x-k)-48 \geq 0
$$

contradicting (18).

Case 1.1.2. $\frac{1}{2} k<y<k-3$.

Then by (24) and (32),

$$
-2(2 y-x)(k-y-3)-2 y^{2}-k x+3 k y-16 x-2 y+17 k-48 \geq 0,
$$


or equivalently,

$$
x-y \leq \frac{17 k-12 y-48}{2 y-k+22} .
$$

Since $y>\frac{1}{2} k,(35)$ implies

$$
x-y<\frac{11 k-48}{22}<\frac{1}{2} k .
$$

Hence by (19) and the hypothesis of Case 1,

$$
5 \leq t<\frac{1}{2} k \text {. }
$$

By (18) and (19), $y=x-t \geq k-t$, so (35) also implies

$$
t \leq \frac{17 k-12(k-t)-48}{2(k-t)-k+22}
$$

or equivalently,

$$
(t-5)(2 t-k)-48 \geq 0
$$

contradicting (36).

Case 1.1.3. $y \geq k-3$.

If $x_{Y Y}=y$, then $x=y$ and $t=0$. This contradicts the hypothesis of Case 1, so we must have $x_{Y Y} \leq y-1$. Hence by (32),

$$
-2(y-1)(k-y-3)-2 y^{2}-k x+3 k y-16 x-2 y+17 k-48 \geq 0,
$$

or equivalently,

$$
x-y \leq \frac{19 k-14 y-54}{k+16} .
$$

Since $y \geq k-3,(37)$ implies

$$
t=x-y \leq \frac{5 k-12}{k+16}<5
$$

contradicting the hypothesis of Case 1 .

Case 1.2. $2 x+y-3 k+7 \leq 0$.

Then by (31),

$$
-x_{Y Y}(2 x-y-k+1)-2 x^{2}+3 x y+2 k x-3 k y-23 x+12 y+17 k-48 \geq 0,
$$

or equivalently,

$$
-x_{Y Y}(2 x-y-k+1)+(x-k)(3 y-2 x)-23 x+12 y+17 k-48 \geq 0 .
$$

Case 1.2.1. $y \leq \frac{1}{2} k$.

Then from (18) and (39) we deduce that

$$
0 \leq-23 x+12 y+17 k-48 \leq-23 x+23 k-48<0,
$$


a contradiction.

Case 1.2.2. $y>\frac{1}{2} k$.

Clearly, $y \leq x$, implying that

$$
2 x-y-k+1 \geq x-k+1>0 .
$$

Hence by (24) and (38),

$$
\begin{aligned}
& -(2 y-x)(2 x-y-k+1)-2 x^{2}+3 x y+2 k x-3 k y-23 x \\
& \quad+12 y+17 k-48 \geq 0
\end{aligned}
$$

which is equivalent to (34). We obtain a contradiction as in Case 1.1.2. (Note that after (34) the assumption $y<k-3$ in the hypothesis of Case 1.1.2 is no longer used.)

Case 2. $t \leq 4$.

Let $S_{1}, \ldots, S_{t}$ be the vertex sets of the components of $C-(X \cup Y)$, so that $S=\cup_{i=1}^{t} S_{i}$. For $i=1, \ldots, t$, let $u_{i}$ be the (unique) vertex of $U$ in $S_{i}$ and $w_{i}$ the vertex of $W$ in $S_{i}$.

Assume $v \in R$. Since $C$ is a nonextendable dominating cycle, $N(v) \subseteq V(C)$ and $N(v) \cap N(v)^{+}=\emptyset$. Furthermore, by Lemma 6, there is at most one value of $i$ for which $N(v) \cap S_{i}$ contains both $u_{i}$ and $w_{i}$. Hence by (18), (19), (20) and the hypothesis of Case 2,

$$
\begin{aligned}
\varepsilon(v, S) & \leq \frac{1}{2}(s+1) \leq \frac{1}{2}\left(\frac{7}{2} k-r-x-y-7\right) \\
& =\frac{1}{2}\left(\frac{7}{2} k-r-2 x+t-7\right) \leq \frac{1}{2}\left(\frac{3}{2} k-r-3\right) .
\end{aligned}
$$

By Lemma $6, \varepsilon(v, Y) \leq 1$. Hence by $(40)$,

$$
\varepsilon(v, X) \geq k-\frac{1}{2}\left(\frac{3}{2} k-r-3\right)-1=\frac{1}{4}(k+2 r+2),
$$

implying that

$$
\varepsilon(R, X) \geq \frac{1}{4} r(k+2 r+2)
$$

Case 2.1. $t \leq 1$.

By (21),

$$
\varepsilon(R, X) \leq k x-k(y+1)+r=k(t-1)+r .
$$

Since $t \leq 1,(41)$ and (42) together imply that $r=0$. Hence if $t=0$, then (42) gives a contradiction right away. If $t=1$, then by (21),

$$
k x \geq \varepsilon(Y \cup\{a\}, X)+\varepsilon\left(\left\{u_{1}, w_{1}\right\}, X\right) \geq k(y+1)+2=k x+2,
$$

again a contradiction.

Case 2.2. $t \in\{2,3,4\}$.

Using Lemmas 5(a) and 6, (18), (19), and (20) we obtain

$$
\varepsilon\left(\left\{u_{1}, u_{2}\right\}, S \cup R\right) \leq s+r \leq \frac{7}{2} k-2 x+t-8 \leq \frac{3}{2} k+t-8,
$$

implying that

$$
\varepsilon\left(\left\{u_{1}, u_{2}\right\}, X\right) \geq 2 k-\left(\frac{3}{2} k+t-8\right)=\frac{1}{2} k+8-t .
$$


Similarly,

$$
\varepsilon\left(\left\{w_{1}, w_{2}\right\}, X\right) \geq \frac{1}{2} k+8-t .
$$

Using (19), (21), (41), (43), and (44) we obtain

$$
\begin{aligned}
k x & \geq \varepsilon(Y \cup\{a\}, X)+\varepsilon(R, X)+\varepsilon\left(\left\{u_{1}, u_{2}, w_{1}, w_{2}\right\}, X\right) \\
& \geq k(y+1)-r+\frac{1}{4} r(k+2 r+2)+k+16-2 t \\
& =k(x-t+2)+\frac{1}{4} r(k+2 r-2)+16-2 t .
\end{aligned}
$$

If $t=2$ this gives a contradiction and hence we have $t \in\{3,4\}$.

For $t=3$ we have by (45),

$$
k x \geq k(x-1)+\frac{1}{4} r(k+2 r-2)+10,
$$

and for $t=4$ we obtain, again using (19), (21), (41), (43), and (44),

$$
\begin{aligned}
k x & \geq \varepsilon(Y \cup\{a\}, X)+\varepsilon(R, X)+\varepsilon\left(\left\{u_{1}, u_{2}, u_{3}, u_{4}, w_{1}, w_{2}, w_{3}, w_{4}\right\}, X\right) \\
& \geq k(y+1)-r+\frac{1}{4} r(k+2 r+2)+2 k+32-4 t \\
& =k(x-1)+\frac{1}{4} r(k+2 r-2)+16 .
\end{aligned}
$$

In both cases we conclude that

$$
r \leq 3 \text {. }
$$

Using Lemmas 5(b) and 6, (18), (19), and (20) we obtain

$$
\begin{aligned}
\varepsilon\left(\left\{u_{1}, u_{2}, u_{3}\right\}, S \cup R\right) & \leq s+t+\left|Z_{U}\right|+r \\
& \leq \frac{7}{2} k-2 x+2 t-8+\left|Z_{U}\right| \leq \frac{3}{2} k+\left|Z_{U}\right|
\end{aligned}
$$

implying that

$$
\varepsilon\left(\left\{u_{1}, u_{2}, u_{3}\right\}, X\right) \geq 3 k-\left(\frac{3}{2} k+\left|Z_{U}\right|\right)=\frac{3}{2} k-\left|Z_{U}\right|
$$

Similarly,

$$
\varepsilon\left(\left\{w_{1}, w_{2}, w_{3}\right\}, X\right) \geq \frac{3}{2} k-\left|Z_{W}\right| .
$$

Assume $z \in Z_{U} \cup Z_{W}$. Using Lemma 5(c), (18), (19), (20), and (46) we obtain

$$
\varepsilon(z, S \cup R) \leq \frac{1}{2} s+r \leq \frac{1}{2}\left(\frac{7}{2} k+r-2 x+t-8\right) \leq \frac{1}{2}\left(\frac{3}{2} k+r-4\right) \leq \frac{1}{4}(3 k-2),
$$

implying that

$$
\varepsilon\left(Z_{U} \cup Z_{W}, X\right) \geq\left|Z_{U} \cup Z_{W}\right|\left(k-\frac{1}{4}(3 k-2)\right)=\frac{1}{4}\left|Z_{U} \cup Z_{W}\right|(k+2) .
$$

Set

$$
T=V(G) \backslash\left(X \cup Y \cup Z_{U} \cup Z_{W} \cup R \cup\left\{a, u_{1}, u_{2}, u_{3}, w_{1}, w_{2}, w_{3}\right\}\right) .
$$

If $t=4$, then $u_{4}, w_{4} \in T$, implying that $\varepsilon(T, X) \geq 2$. Hence for both $t=3$ and $t=4$,

$$
\varepsilon(T, X) \geq 2(t-3) .
$$


From (17), (21), (41), (47), (48), (49), and (50) we obtain

$$
\begin{aligned}
k x \geq & \varepsilon(Y \cup\{a\}, X)+\varepsilon(R, X)+\varepsilon\left(\left\{u_{1}, u_{2}, u_{3}\right\}, X\right)+\varepsilon\left(\left\{w_{1}, w_{2}, w_{3}\right\}, X\right) \\
& +\varepsilon\left(Z_{U} \cup Z_{W}, X\right)+\varepsilon(T, X) \geq k(y+1)-r+\frac{1}{4} r(k+2 r+2)+3 k \\
& -\left|Z_{U}\right|-\left|Z_{W}\right|+\frac{1}{4}\left|Z_{U} \cup Z_{W}\right|(k+2)+2(t-3) \geq k(y+1)+3 k+2(t-3) \\
= & k x+k(4-t)+2(t-3)>k x,
\end{aligned}
$$

our final contradiction.

\section{PROOF OF THEOREM 7}

For the proof of Theorem 7 we need some additional terminology and notation.

Let $\lambda$ be a positive integer. A cycle $C$ of a graph $G$ is called a $D_{\lambda}$-cycle if every component of $G-V(C)$ has order smaller than $\lambda$. Hence a $D_{1}$-cycle is a Hamilton cycle and a $D_{2}$-cycle is a dominating cycle.

Let $X, X_{1}, X_{2}$ be subgraphs of $G$. By $N(X)$ we denote the set $\{v \in V(G) \backslash V(X) \mid \varepsilon(v, X)$ $>0\}$. The subgraphs $X_{1}$ and $X_{2}$ are called remote if $V\left(X_{1}\right) \cap V\left(X_{2}\right)=N\left(X_{1}\right) \cap V\left(X_{2}\right)=$ $\emptyset$. The number of components of $X$ with at least $\lambda$ vertices is denoted by $\omega_{\lambda}(X)$.

If $\vec{C}$ is a cycle of $G$ and $v \in V(C)$, then a subgraph $X$ of $G$ is called a $(\vec{C}, v, \lambda)$-subgraph if each of the following conditions is satisfied:

(i) $X$ is connected and has order $\lambda$;

(ii) $V(X) \cap V(C)=v \vec{C} w$ for some vertex $w \in V(C)$;

(iii) if $X^{\prime}$ satisfies (i) and (ii), then $V(X) \cap V(C) \subseteq V\left(X^{\prime}\right) \cap V(C)$.

Proof of Theorem 7. Let $G$ be a 2-connected $k$-regular graph of order $n \leq 4 k-3$ and assume $G$ contains no dominating cycle. Set

$$
\lambda+1=\min \left\{i \mid G \text { has a } D_{i} \text {-cycle }\right\}
$$

so that $\lambda \geq 2$. Let $\vec{C}$ be a $D_{\lambda+1}$-cycle of $G$ for which $\omega_{\lambda}(G-V(C))$ is minimum. Since $G$ has no $D_{\lambda}$-cycle, $G-V(C)$ has a component $X_{0}$ of order $\lambda$. Let $x_{1}, \ldots, x_{m}$ be the vertices in $N\left(X_{0}\right)$, occurring on $\vec{C}$ in the order of their indices. As in the proof of [11, Theorem 2] there exists, for $i=1, \ldots, m$, a $\left(\vec{C}, x_{i}^{+}, \lambda\right)$-subgraph $X_{i}$ such that $X_{0}, X_{1}, \ldots, X_{m}$ are pairwise remote. Set $R=V(G) \backslash \cup_{i=0}^{m} V\left(X_{i}\right)$. Since $G$ is $k$-regular and $X_{0}, X_{1}, \ldots, X_{m}$ are pairwise remote, we have

$$
\varepsilon(v, R) \geq k-\lambda+1 \quad \text { for all } v \in \bigcup_{i=0}^{m} V\left(X_{i}\right) .
$$

We distinguish two cases. In the first case we reach a contradiction, while in the second we conclude that $G \in \mathcal{F}$.

Case 1. $2 \leq \lambda \leq k-2$.

By (51), $m \geq k-\lambda+1$ and

$$
k|R| \geq \varepsilon\left(\bigcup_{i=0}^{m} V\left(X_{i}\right), R\right) \geq(m+1) \lambda(k-\lambda+1) \geq(k-\lambda+2) \lambda(k-\lambda+1),
$$


implying that

$$
k(4 k-3) \geq k n=k(m+1) \lambda+k|R| \geq \lambda(k-\lambda+2)(2 k-\lambda+1) .
$$

Set $\alpha=\lambda-2$ and $\beta=k-\lambda-2$. Then $\lambda=\alpha+2$ and $k=\alpha+\beta+4$, so

$$
\begin{aligned}
& \lambda(k-\lambda+2)(2 k-\lambda+1)-k(4 k-3) \\
& \quad=(\alpha+2)(\beta+4)(\alpha+2 \beta+7)-(\alpha+\beta+4)(4 \alpha+4 \beta+13) \\
& \quad=\alpha^{2} \beta+2 \alpha \beta^{2}+9 \alpha \beta+7 \alpha+\beta+4>0,
\end{aligned}
$$

contradicting (52).

Case 2. $\lambda \geq k-1$.

Since $\left\{x_{1}, \ldots, x_{m}\right\} \subseteq R$, we have

$$
4 k-3 \geq n \geq(m+1) \lambda+|R| \geq(m+1) \lambda+m .
$$

If $m \geq 3$, then by (53), $4 k-3 \geq 4(k-1)+3$, a contradiction. Since $G$ is 2 -connected, we conclude that

$$
m=2 .
$$

If $\lambda=k-1$, then by (51),

$$
\begin{aligned}
2 k & \geq \varepsilon\left(\left\{x_{1}, x_{2}\right\}, V\left(X_{0}\right) \cup\left\{x_{1}^{-}, x_{1}^{+}, x_{2}^{-}, x_{2}^{+}\right\}\right) \geq \varepsilon\left(V\left(X_{0}\right),\left\{x_{1}, x_{2}\right\}\right)+4 \\
& \geq \lambda(k-\lambda+1)+4=2 k+2,
\end{aligned}
$$

a contradiction. Hence

$$
\lambda \geq k
$$

By (53), (54), and (55),

$$
4 k-3 \geq 3 \lambda+2 \geq \lambda+2 k+2,
$$

implying that $\left|V\left(X_{0}\right)\right|=\lambda \leq 2 k-5$. Since $\delta\left(X_{0}\right) \geq k-m=k-2$, it follows that $\delta\left(X_{0}\right) \geq \frac{1}{2}\left(\left|V\left(X_{0}\right)\right|+1\right)$. Hence $X_{0}$ is Hamilton-connected, i.e., every pair of vertices in $X_{0}$ is joined by a Hamilton path of $X_{0}$ (see, e.g., [1]). Thus there exists a cycle of $G$ that properly contains all vertices of $X_{0}$; in each such cycle $C^{\prime}$, the subgraph of $C^{\prime}$ induced by the vertices of $X_{0}$ is a path. Now set

$$
\mu+1=\min \left\{i \mid G \text { has a } D_{i} \text {-cycle containing } V\left(X_{0}\right)\right\} .
$$

Clearly, $\mu \geq \lambda$. Among all $D_{\mu+1}$-cycles containing $V\left(X_{0}\right)$, let $\vec{C}_{1}$ be one for which $\omega_{\mu}(G-$ $\left.V\left(C_{1}\right)\right)$ is minimum. Let $Y_{0}$ be a component of $G-V\left(C_{1}\right)$ of order $\mu$ and $y_{1}, \ldots, y_{p}$ the vertices of $N\left(Y_{0}\right)$, occurring on $\vec{C}_{1}$ in the order of their indices such that $V\left(X_{0}\right) \subseteq y_{1}^{+} \vec{C}_{1} y_{2}^{-}$. By the argument in the proof of [11, Theorem 2] there exists, for $i=2, \ldots, p, \mathbf{a}\left(\vec{C}_{1}, y_{i}^{+}, \mu\right)-$ subgraph $Y_{i}$ such that $Y_{0}, Y_{2}, \ldots, Y_{p}$ are pairwise remote. Hence

$$
4 k-3 \geq n \geq\left|V\left(X_{0}\right)\right|+\left|V\left(Y_{0}\right)\right|+\left|\bigcup_{i=2}^{p} V\left(Y_{i}\right)\right|+\left|N\left(Y_{0}\right)\right|=\lambda+p \mu+p .
$$


Arguing as for $X_{0}$ we obtain $\left|V\left(Y_{0}\right)\right|=\mu \leq 2 k-5$ and $\delta\left(Y_{0}\right) \geq k-p=k-2$, implying that $Y_{0}$ is Hamilton-connected. Thus there exists a cycle of $G$ containing $V\left(X_{0}\right) \cup V\left(Y_{0}\right)$. Set

$$
\nu+1=\min \left\{i \mid G \text { has a } D_{i} \text {-cycle containing } V\left(X_{0}\right) \cup V\left(Y_{0}\right)\right\} .
$$

Clearly, $\nu \geq \mu$. Among all $D_{\nu+1}$-cycles containing $V\left(X_{0}\right) \cup V\left(Y_{0}\right)$, let $\vec{C}_{2}$ be one for which $\omega_{\nu}\left(G-V\left(C_{2}\right)\right)$ is minimum. Let $Z_{0}$ be a component of $G-V\left(C_{2}\right)$ of order $\nu$ and $z_{1}, \ldots, z_{q}$ the vertices in $N\left(Z_{0}\right)$, occurring on $\vec{C}_{2}$ in the order of their indices such that $V\left(X_{0}\right) \subseteq z_{1}^{+} \vec{C}_{2} z_{2}^{-}$. If $z_{i}^{+} \vec{C}_{2} z_{i+1}^{-} \cap V\left(Y_{0}\right)=\emptyset$ for some $i \in\{2, \ldots, q\}$ (indices $\bmod q$ ), then there exists a $\left(\vec{C}_{2}, z_{i}^{+}, \nu\right)$-subgraph $Z_{i}$ such that $Z_{0}$ and $Z_{i}$ are remote, implying that

$$
4 k-3 \geq n>\left|V\left(X_{0}\right)\right|+\left|V\left(Y_{0}\right)\right|+\left|V\left(Z_{0}\right)\right|+\left|V\left(Z_{i}\right)\right|=\lambda+\mu+2 \nu \geq 4 k,
$$

a contradiction. It follows that $q=2$ and $V\left(Y_{0}\right) \subseteq z_{2}^{+} \vec{C}_{2} z_{1}^{-}$. Set $S=\left\{x_{1}, x_{2}, y_{1}, y_{2}, z_{1}, z_{2}\right\}$ and $T=V(G) \backslash\left(V\left(X_{0}\right) \cup V\left(Y_{0}\right) \cup V\left(Z_{0}\right) \cup S\right)$. (Note that $\left\{x_{1}, x_{2}\right\},\left\{y_{1}, y_{2}\right\},\left\{z_{1}, z_{2}\right\}$ need not be pairwise disjoint; the three sets may even coincide.) We have

$$
|T|=n-\lambda-\mu-\nu-|S| \leq 4 k-3-3 k-|S|=k-|S|-3,
$$

implying that

$$
|T||S| \geq \varepsilon(T, S)=\varepsilon(T, V(G) \backslash T) \geq|T|(k-|T|+1) \geq|T|(|S|+4) .
$$

It follows that $T=\emptyset$. Since $Z_{0}$, like $X_{0}$ and $Y_{0}$, is Hamilton-connected while $G$ is nonhamiltonian, we now easily conclude that $G \in \mathcal{F}$.

Remark. It follows from the remark after Theorem 3 in Section 1 that Theorem 7 is close to the best possible result. We did not attempt to increase the bound on $|V(G)|$ given in Theorem 7 beyond $4 k-3$ in this paper, since the present result is already more than is needed for our main result, Theorem 5 , and its proof is relatively simple. Using additional and more complicated arguments, the fourth author has now convinced himself that, for $k$ sufficiently large, the bound $4 k-3$ in Theorem 7 can be improved to $4 k+3$. The resulting theorem is best possible: for $k \geq 4$, the graph $\overline{K_{3}} \vee\left(K_{k-2}+3 K_{k+1}\right)$ of order $4 k+4$ has a (unique) 2-connected (but not 3-connected) $k$-regular spanning subgraph, which contains no dominating cycle and does not belong to $\mathcal{F}$.

\section{References}

[1] J. A. Bondy and V. Chvátal, A method in graph theory, Discrete Math. 15 (1976), 111-135.

[2] J. A. Bondy and M. Kouider, Hamilton cycles in regular 2-connected graphs, J. Combin. Theory B 44 (1988), 177-186.

[3] J. A. Bondy and U. S. R. Murty, Graph Theory with Applications, Macmillan, London and Elsevier, New York (1976).

[4] H. J. Broersma, J. van den Heuvel, H. A. Jung, and H. J. Veldman, Cycles containing all vertices of maximum degree, J. Graph Theory 17 (1993), 373-385.

[5] H. J. Broersma, J. van den Heuvel, and H. J. Veldman, A generalization of Ore's Theorem involving neighborhood unions, Discrete Math. 122 (1993), 37-49. 
[6] G. A. Dirac, Some theorems on abstract graphs, Proc. London Math. Soc. (3) 2 (1952), 69-81.

[7] F. Hilbig, Kantenstrukturen in nichthamiltonschen Graphen. Ph.D. Thesis, Technische Universität Berlin (1986).

[8] B. Jackson, Hamilton cycles in regular 2-connected graphs, J. Combin. Theory B 29 (1980), $27-46$.

[9] B. Jackson, H. Li, and Y. Zhu, Dominating cycles in regular 3-connected graphs, Discrete Math. 102 (1991), 163-176.

[10] Min Aung, Circumference of a regular graph, J. Graph Theory 13 (1989), 149-155.

[11] H. J. Veldman, Existence of $D_{\lambda}$-cycles and $D_{\lambda}$-paths, Discrete Math. 44 (1983), 309-316.

[12] M. E. Watkins and D. M. Mesner, Cycles and connectivity in graphs, Can. J. Math. 19 (1967), 1319-1328.

[13] D. R. Woodall, The binding number of a graph and its Anderson number, J. Combin. Theory B 15 (1973), 225-255.

[14] Y. Zhu and H. Li, Hamilton cycles in regular 3-connected graphs, Discrete Math. 110 (1992), 229-249.

[15] Y. Zhu, Z. Liu, and Z. Yu, 2-Connected $k$-regular graphs on at most $3 k+3$ vertices to be hamiltonian, J. Sys. Sci. \& Math. Sci. 6 (1) (1986), 36-49; (2) (1986), 136-145.

Received June 15, 1994 